\title{
The Exact Order of Convergence for Finite Difference Approximations to Ordinary Boundary Value Problems
}

\author{
By Wolf-Jürgen Beyn
}

\begin{abstract}
This paper deals with the problem of determining the exact order of convergence for the finite difference method applied to ordinary boundary value problems when formulas of different orders are used at different points of the grid. Under rather general assumptions, it is shown that the global discretization error is $O\left(h^{\tau}\right)$ if the local truncation error is $O\left(h^{\tau}\right)$ on the boundary and at interior grid points, while it is only $O\left(h^{\tau-(k-\mu)}\right)$ at grid points near the boundary. Here $k$ and $\mu$ denote the order of the differential and the boundary operator, respectively.
\end{abstract}

1. Introduction. For finite difference methods it is a standard procedure to conclude convergence from consistency and stability. Moreover, the order of convergence is shown to be at least the order of consistency (cf. Stetter [16]).

However, Bramble and Hubbard have shown in a series of papers [3]- [5] (see also Price [15]) that the order of convergence may be improved in special cases. For various finite difference approximations to ordinary and partial boundary value problems they established the following rule: The order of convergence is $\tau$ if the order of consistency is $\tau$ for the boundary conditions and

$\left.\begin{array}{l}\tau \text { at interior grid points, } \\ \tau-(k-\mu) \text { at grid points near the boundary }\end{array}\right\}$ for the differential equation, where $k$ and $\mu$ denote the order of the differential equation and the boundary conditions, respectively.

In order to obtain this rule it is evident that instead of the usual stability inequalities one needs weighted stability inequalities (see Section 2). These are derived in [4], [5] from the monotone properties of the difference equations and from the existence of appropriate grid functions (for a systematic approach see Ciarlet [6]). For a more elegant method, which avoids the construction of special grid functions, we refer to Lorenz [13], [14]. All of these results basically require that the difference equations lead to an inverse monotone matrix. Hence, they are applicable to ordinary as well as partial boundary value problems but are mainly restricted to secondorder equations which are inverse monotone (i.e., which have a nonnegative Green's function).

The first general result for the ordinary finite difference method is due to

Received September 7, 1977.

AMS (MOS) subject classifications (1970). Primary 65 L10. 
Kreiss [12] (see Esser [7] for an extension) who obtained the above rule in the cases $\mu=k-1$ and $\mu=k-2$, but his method seems not to generalize directly (cf. Section 2).

In this paper we shall prove the rule of Bramble and Hubbard in the ordinary case under rather general assumptions. We consider an $m$-dimensional system of ordinary boundary value problems of order $k$

$$
L x:=\sum_{i=0}^{k} p_{i} x^{(i)}=r \quad \text { in }[a, b], \quad R x=\gamma,
$$

where $x \in\left(C^{k}[a, b]\right)^{m}, r \in(C[a, b])^{m}, \gamma \in \mathbf{R}^{m k}, p_{i}(t), t \in[a, b], i=0, \ldots, k$, are continuous $m \times m$ matrices, $p_{k}(t)$ is nonsingular for all $t \in[a, b]$ and $R:\left(C^{k-1}[a, b]\right)^{m} \rightarrow \mathbf{R}^{m k}$ is a linear mapping.

Let $H$ be a real positive sequence converging to zero. For every mesh size $h \in H$ we approximate (1) by finite difference equations

$$
L_{h} x_{h}=r_{h}, \quad R_{h} x_{h}=\gamma,
$$

where $x_{h}$ and $r_{h}$ are grid functions and the pairs $\left(L_{h}, R_{h}\right)$ are discrete analogues of $(L, R)$ (see Section 2 for details).

In Section 2 we derive weighted stability inequalities for the pairs $\left(L_{h}, R_{h}\right)$ by means of stability itself and an additional condition for the difference operator $L_{h}$.

Furthermore, using the stability theory of [12], [1], we shall show that both assumptions are satisfied if certain root conditions hold for the principal part of the difference operator $L_{h}$ (see Sections 3 and 4). These root conditions have been verified in [1] for a wide class of difference approximations.

Throughout our analysis we use maximum norms of difference quotients over the given grid. Therefore, we also obtain a modified rule which determines the order of convergence of the difference quotients, or of certain difference formulas, applied to the solution of the discrete system (2). This will be illustrated in Section 5 by some numerical examples.

Finally, it will be shown that the weighted stability inequalities derived in Sections 2, 3 and 4 cannot, in general, be improved.

2. Weighted Stability Inequalities. Let us briefly describe the difference equations (2) and introduce some notation.

For $h \in H$ let $J_{h}=\left\{a_{h}, a_{h}+h, \ldots, b_{h}-h, b_{h}\right\} \subset \mathbf{R}$ be the underlying equidistant grid, where the endpoints $a_{h}, b_{h}$ satisfy

$$
h^{-1}\left(b_{h}-a_{h}\right) \in \mathbf{N}, \quad a_{h} \rightarrow a \text { and } b_{h} \rightarrow b \text { for } h \rightarrow 0(h \in H) .
$$

Furthermore, let $J_{h}^{\prime}=\left\{a_{h}^{\prime}=a_{h}+k_{1} h, a_{h}^{\prime}+h, \ldots, b_{h}^{\prime}-h, b_{h}^{\prime}=b_{h}-k_{2} h\right\}$, where $k_{1}, k_{2} \in \mathrm{N}$ and $k_{1}+k_{2}=k$. By $X_{h}$ and $X_{h}^{\prime}$ we denote the space of grid functions with values in $\mathbf{R}^{m}$ and with domains $J_{h}$ and $J_{h}^{\prime}$, respectively. We assume $x_{h} \in X_{h}$, $r_{h} \in X_{h}^{\prime}$ and $L_{h}: X_{h} \rightarrow X_{h}^{\prime}, R_{h}: X_{h} \rightarrow \mathbf{R}^{m k}$ are linear operators, so that (2) constitutes a system of $m\left|J_{h}\right|$ linear equations in $m\left|J_{h}\right|$ unknowns $\left(\left|J_{h}\right|=h^{-1}\left(b_{h}-a_{h}\right)+1\right)$. 
Nearly every reasonable difference equation which approximates (1) on an equidistant grid may be written in the form (2). We consider as an example the following Sturm-Liouville boundary value problem:

$$
x^{\prime \prime}+p_{0} x=r \quad \text { in }[a, b], \quad x^{\prime}(a)=\gamma_{a}, \quad x(b)=\gamma_{b},
$$

and the difference equations

$$
\begin{aligned}
& r(t)=p_{0}(t) x_{h}(t)+\left\{\begin{array}{r}
h^{-2}\left(x_{h}(t-h)-2 x_{h}(t)+x_{h}(t+h)\right) \\
\text { for } t=a \text { and } t=b-h, \\
\left(12 h^{2}\right)^{-1}\left(-x_{h}(t-2 h)+16 x_{h}(t-h)-30 x_{h}(t)\right. \\
\left.+16 x_{h}(t+h)-x_{h}(t+2 h)\right) \\
\text { for } t=a+h, \ldots, b-2 h,
\end{array}\right. \\
& \gamma_{a}=(6 h)^{-1}\left(-2 x_{h}(a-h)-3 x_{h}(a)+6 x_{h}(a+h)-x_{h}(a+2 h)\right) \\
& \gamma_{b}=x_{h}(b),
\end{aligned}
$$

where $h=(b-a) N^{-1}, N \geqslant 4$. With $a_{h}=a-h, b_{h}=b, k_{1}=1, k_{2}=1$ the system (5), (6) may obviously be written in the form (2). The difference formulas used for $x^{\prime \prime}(t)$ are of order 2 at $t=a, b-h$ and of order 4 at $t=a+h, \ldots, b-2 h$, while the formula for $x^{\prime}(a)$ is of order 3 .

For a grid function $x_{h}: K_{h} \rightarrow \mathbf{R}^{m}$ with domain $K_{h} \subset J_{h}$ we set

$$
\begin{aligned}
& E^{\nu} x_{h}(t)=x_{h}(t+\nu h) \quad \text { if } t+\nu h \in K_{h}, \nu \in \mathbf{Z} \text { and } \\
& \Delta^{\nu} x_{h}(t)=h^{-\nu}(E-I)^{\nu} x_{h}(t) \quad \text { if } t+\mu h \in K_{h}(\mu=0, \ldots, \nu), \nu \in \mathbf{N} .
\end{aligned}
$$

Furthermore, we define

(7) $\left\|x_{h}\right\|_{j, M_{h}}=\sum_{\nu=0}^{j} \operatorname{Max}\left\{\left|\left(\Delta^{\nu} x_{h}(t)\right)_{i}\right|: i=1, \ldots, m, t+\mu h \in M_{h}(\mu=0, \ldots, \nu)\right\}$,

where $j \in \mathbf{N}$ and $M_{h}$ is any subset of the domain $K_{h}$. In case $M_{h}=K_{h}$ we write \|\|$_{j}=\|\|_{j, M_{h}}$. \|\|$_{0}$ will also be used to denote the maximum norm in $\mathbf{R}^{n}, n \in \mathbf{N}$.

A sequence of linear operators $L_{h}: X_{h} \rightarrow X_{h}^{\prime}(h \in H)$ or $R_{h}: X_{h} \rightarrow \mathbf{R}^{m k}(h \in H)$ is called \|\|$_{j}$-bounded if there exists a constant $C>0$ such that $\left\|L_{h} x_{h}\right\|_{0} \leqslant C\left\|x_{h}\right\|_{j}$ or $\left\|R_{h} x_{h}\right\|_{0} \leqslant C\left\|x_{h}\right\|_{j}$ holds for all $x_{h} \in X_{h}, h \in H$.

The minimal $j$ having this property is usually equal to the order of the differential operator $L$ (the boundary operator $R$ ) which is approximated by $L_{h}\left(R_{h}\right)$.

For example, it is easily seen for our difference equations (5), (6) that $L_{h}$ is \|\|$_{2}$-bounded and $R_{h}$ is \|\|$_{1}$-bounded.

The pairs $\left(L_{h}, R_{h}\right)(h \in H)$ are called \|\|$_{j}$-stable if a stability inequality

$$
\left\|x_{h}\right\|_{j} \leqslant C\left(\left\|L_{h} x_{h}\right\|_{0}+\left\|R_{h} x_{h}\right\|_{0}\right) \quad \forall x_{h} \in X_{h}
$$

holds for almost every $h \in H$ and for some constant $C>0$.

Let $[A, B]$ be a real interval containing $[a, b]$ and $J_{h}$ for all $h \in H$ (such an interval exists by (3)) and let $[x]_{h} \in X_{h}$ and $[x]_{h}^{\prime} \in X_{h}^{\prime}$ denote the restrictions of a 
function $x:[A, B] \rightarrow \mathbf{R}^{m}$ to $J_{h}$ and $J_{h}^{\prime}$, respectively. Furthermore, let us extend the coefficients of $L$ continuously to $[A, B]$, then $L_{h}\left(R_{h}\right)$ is called consistent with $L(R)$ if $\left\|L_{h}[x]_{h}-[L x]_{h}^{\prime}\right\|_{0} \rightarrow 0\left(\left\|R_{h}[x]_{h}-R x\right\|_{0} \rightarrow 0\right)$ holds for all $x \in\left(C^{k}[A, B]\right)^{m}$. In view of (3) this definition turns out to be independent of the continuous extension chosen above.

We now assume that, for each $h, J_{h}^{\prime}$ is decomposed into two disjoint subsets, $J_{h}^{0}$ and $J_{h}^{1}$, on which $L_{h}$ has different orders of consistency with respect to the solution $\bar{x}$ of (1), e.g. $\left\|L_{h}[\bar{x}]_{h}-[L \bar{x}]_{h}^{\prime}\right\|_{0, J_{h}^{0}}=O\left(h^{\tau}\right)$ and $\left\|L_{h}[\bar{x}]_{h}-[L \bar{x}]_{h}^{\prime}\right\|_{0, J_{h}^{1}}=O\left(h^{\tau-\rho}\right)$ for some $\rho \in[0, \tau]$. Furthermore, let $\left\|R \bar{x}-R_{h}[\bar{x}]_{h}\right\|_{0}=O\left(h^{\tau}\right)$. Suppose that the following weighted stability inequality is valid

$$
\left\|x_{h}\right\|_{0} \leqslant C\left(\left\|L_{h} x_{h}\right\|_{0, J_{h}^{0}}+h^{\rho}\left\|L_{h} x_{h}\right\|_{0, J_{h}^{1}}+\left\|R_{h} x_{h}\right\|_{0}\right) \quad \forall x_{h} \in X_{h} .
$$

Then (2) has a unique solution $\bar{x}_{h}$, and if $r_{h}=[r]_{h}^{\prime}$ we obtain

$$
\begin{aligned}
\left\|[\bar{x}]_{h}-\bar{x}_{h}\right\|_{0} \leqslant C\left(\left\|L_{h}[\bar{x}]_{h}-[L \bar{x}]_{h}^{\prime}\right\|_{0, J_{h}^{0}}+h^{\rho}\left\|L_{h}[\bar{x}]_{h}-[L \bar{x}]_{h}^{\prime}\right\|_{0, J_{h}^{1}}\right. & \left.+\left\|R_{h}[\bar{x}]_{h}-R \bar{x}\right\|_{0}\right) \\
& =O\left(h^{\tau}\right),
\end{aligned}
$$

which is the desired order of convergence.

Thus, the exponent $\rho$ in inequality (9) indicates precisely how the order of consistency in $J_{h}^{1}$ may be reduced without affecting the order of convergence. Our further study will, therefore, be devoted to weighted stability inequalities.

Theorem 1. Let $\left(L_{h}, R_{h}\right)$ be \|\|$_{j}$-stable and $R_{h}$ be \|\|$_{\mu}$-bounded for some $j, \mu \in\{0, \ldots, k\}$. Assume $J_{h}^{\prime}=J_{h}^{0} \cup J_{h}^{1}, J_{h}^{0}$ and $J_{h}^{1}$ being disjoint, and let $L_{h}$ have the property:

(V) For each $y_{h} \in X_{h}^{\prime}$, satisfying $y_{h}=0$ in $J_{h}^{0}$, there exists $z_{h} \in X_{h}$ such that $L_{h} z_{h}=y_{h},\left\|z_{h}\right\|_{0} \leqslant C h^{k}\left\|y_{h}\right\|_{0}$ with $C>0$ independent of $y_{h}$ and $h \in H$. Then the weighted stability inequality

$$
\left\|x_{h}\right\|_{j} \leqslant C\left(\left\|L_{h} x_{h}\right\|_{0, J_{h}^{0}}+h^{k-\operatorname{Max}(\mu, j)}\left\|L_{h} x_{h}\right\|_{0, J_{h}^{1}}+\left\|R_{h} x_{h}\right\|_{0}\right)
$$

holds for all $x_{h} \in X_{h}$ and almost every $h \in H$.

Proof. For $x_{h} \in X_{h}$ define $y_{h} \in X_{h}^{\prime}$ by

$$
y_{h}= \begin{cases}L_{h} x_{h} & \text { in } J_{h}^{1}, \\ 0 & \text { in } J_{h}^{0} .\end{cases}
$$

Let $z_{h} \in X_{h}$ be given by (V), then we have $\left\|z_{h}\right\|_{i} \leqslant C h^{k-i}\left\|y_{h}\right\|_{0}$ for all $h \in H$ and $i=0, \ldots, k$. Hence, by our assumptions,

$$
\begin{aligned}
\left\|x_{h}\right\|_{j} & \leqslant\left\|x_{h}-z_{h}\right\|_{j}+\left\|z_{h}\right\|_{j} \leqslant C\left\{\left\|L_{h} x_{h}-L_{h} z_{h}\right\|_{0}+\left\|R_{h} x_{h}-R_{h} z_{h}\right\|_{0}+\left\|z_{h}\right\|_{j}\right\} \\
& \leqslant C\left\{\left\|L_{h} x_{h}\right\|_{0, J_{h}^{0}}+\left\|R_{h} x_{h}\right\|_{0}+\left\|z_{h}\right\|_{\mu}+\left\|z_{h}\right\|_{j}\right\} \\
& \leqslant C\left\{\left\|L_{h} x_{h}\right\|_{0, J_{h}^{0}}+\left\|R_{h} x_{h}\right\|_{0}+h^{k-\operatorname{Max}(\mu, j)}\left\|y_{h}\right\|_{0}\right\} .
\end{aligned}
$$


Since $\left\|y_{h}\right\|_{0}=\left\|L_{h} x_{h}\right\|_{0, J_{h}^{1}}$, our proof is finished. Q.E.D.

Theorem 1 generalizes a result of Kreiss [12], who has obtained the inequality (10) in the cases $j=0$ and $\mu=k-1$ or $\mu=k-2$. His proof in the case $\mu=k-1$ essentially uses a property similar to $(\mathrm{V})$, where $L_{h}$ is replaced by its discrete principal part (compare Section 3) and terms of lower order are treated as perturbations.

These results have been extended by Esser [7] to the cases $j=\mu=k-1$ and $j=\mu=k-2$ where, in addition, a discrete $L_{1}$-norm was used on the right-hand side of inequality (10). For $j=0$ Theorem 1 shows that inequality (9) holds with $\rho=$ $k-\mu$ and, if $J_{h}^{1}$ consists of grid points near the boundary, we obtain the rule of Bramble and Hubbard as described in the introduction. If $\left(L_{h}, R_{h}\right)$ is \|\|$_{k}$-stable then inequality (10) is even valid for all $j \in\{0, \ldots, k\}$. We shall investigate this case in more detail, since \|\|$_{k}$-stability and condition (V) will be satisfied under nearly the same assumptions.

3. The Condition (V). The \|\|$_{k}$-stability of pairs $\left(L_{h}, R_{h}\right)$ can be completely characterized if $L_{h}$ can be represented as $L_{h}=p_{k}^{h} E^{-k} \Delta^{k}+L_{h}^{\prime}$, where $p_{k}^{h}: X_{h}^{\prime} \rightarrow X_{h}^{\prime}$ and $L_{h}^{\prime}: X_{h} \rightarrow X_{h}^{\prime}$ are linear operators such that $p_{k}^{h} E^{-k_{1}} \Delta^{k}$ and $L_{h}^{\prime}$ are consistent with $p_{k} D^{k}$ and $L^{\prime}=\sum_{j=0}^{k-1} p_{j} D^{j}$, respectively. In other words, we assume that the principal part of the differential equation has a discrete analogue which may be written as a linear combination of the divided differences $E^{-k} \Delta^{k}$ at different points of the grid. Here $E^{-k} \Delta^{k}$ is considered as a linear operator mapping $X_{h}$ into $X_{h}^{\prime}$. Such a decomposition is natural for common finite difference schemes (cf. [1], [8], [9], [17]).

For our example (5) we have $L_{h}^{\prime} x_{h}(t)=p_{0}(t) x_{h}(t), t \in J_{n h}^{\prime}, x_{h} \in X_{h}$, and

$$
p_{2}^{h} y_{h}(t)=\left\{\begin{array}{l}
y_{h}(t) \quad \text { for } t=a, b-h, \\
\frac{1}{12}\left(-y_{h}(t-h)+14 y_{h}(t)-y_{h}(t+h)\right) \text { for } t=a+h, \ldots, b-2 h,
\end{array}\right.
$$

where $y_{h} \in X_{h}^{\prime}$.

For a proof of the following theorem we refer to [1] (see [8], [9] , [12] for related theorems).

Theorem 2. Assume that 0 is not an eigenvalue of $\left(L=p_{k} D^{k}+L^{\prime}, R\right)$. Let $p_{k}^{h} E^{-k} \Delta^{k}, L_{h}^{\prime}$ and $R_{h}$ be consistent with $p_{k} D^{k}, L^{\prime}$ and $R$, respectively, and let $L_{h}^{\prime}$ be \|\|$_{k-1}$-bounded and $R_{h}$ be \|\|$_{k}$-bounded. Then $\left(L_{h}, R_{h}\right)$ is \|\|$_{k}$-stable if and only if $p_{k}^{h}$ is \|\|$_{0}$-stable, i.e., there exists a constant $C>0$ such that

$$
\left\|x_{h}\right\|_{0} \leqslant C\left\|p_{k}^{h} x_{h}\right\|_{0} \quad \forall x_{h} \in X_{h}^{\prime} \text { and for almost every } h \in H \text {. }
$$

Using the same representation of $L_{h}$ as above we are going to prove condition $(\mathrm{V})$, where $J_{h}^{1}$ is now assumed to consist of a finite number of grid points near the boundary. Let $J_{h}^{1}=\left\{a_{h}^{\prime}, \ldots, a_{h}^{\prime}+n_{a} h, b_{h}^{\prime}-n_{b} h, \ldots, b_{h}^{\prime}\right\}$ and $J_{h}^{0}=\left\{a_{h}^{\prime}+\right.$ $\left.\left(n_{a}+1\right) h, \ldots, b_{h}^{\prime}-\left(n_{b}+1\right) h\right\}$ with $n_{a}, n_{b} \in \mathbf{N}$ independent of $h$. This will be the interesting case with applications. 
For a given $y_{h} \in X_{h}^{\prime}$ that vanishes in $J_{h}^{0}$ we write $y_{h}=y_{h}^{a}+y_{h}^{b}$, where

$$
\begin{aligned}
& y_{h}^{a}(t)= \begin{cases}y_{h}(t) & \text { for } t=a_{h}^{\prime}, \ldots, a_{h}^{\prime}+n_{a} h, \\
0 & \text { otherwise, }\end{cases} \\
& y_{h}^{b}(t)= \begin{cases}y_{h}(t) & \text { for } t=b_{h}^{\prime}-n_{b} h, \ldots, b_{h}^{\prime}, \\
0 & \text { otherwise. }\end{cases}
\end{aligned}
$$

It is obviously sufficient to prove the condition (V) for $y_{h}^{a}$ and $y_{h}^{b}$ separately. We shall construct the corresponding $z_{h}^{a}, z_{h}^{b} \in X_{h}$ as solutions of the following discrete initial-value problems

$$
\begin{gathered}
L_{h} z_{h}^{a}=y_{h}^{a}, \quad \Delta^{v} z_{h}^{a}\left(b_{h}-(k-1) h\right)=0 \quad \text { for } \nu=0, \ldots, k-1, \\
L_{h} z_{h}^{b}=y_{h}^{b}, \quad \Delta^{v} z_{h}^{b}\left(a_{h}\right)=0 \quad \text { for } v=0, \ldots, k-1 .
\end{gathered}
$$

Note that the matrices associated with the systems (12) and (13) are not usually of triangular form. Thus, we cannot expect $z_{h}^{a}$ or $z_{h}^{b}$ to vanish on the right-hand or lefthand side of $J_{h}$. But the behavior of $z_{h}^{a}$ and $z_{h}^{b}$ may be well described by means of the "boundary layer functions"

$$
e(t)=\sigma^{h^{-1}\left(b_{h}^{\prime}-t\right)}, \quad \bar{e}(t)=\sigma^{h^{-1}\left(t-a_{h}^{\prime}\right)}, \quad t \in J_{h}(0<\sigma<1),
$$

and the corresponding weighted maximum norms

$$
\begin{aligned}
\left\|x_{h}\right\|_{e} & =\operatorname{Max}\left\{\left|x_{h}(t)\right|_{i}(e(t))^{-1}: i=1, \ldots, m, t \in K_{h}\right\}, \\
\left\|x_{h}\right\|_{\bar{e}} & =\operatorname{Max}\left\{\left|x_{h}(t)\right|_{i}(\bar{e}(t))^{-1}: i=1, \ldots, m, t \in K_{h}\right\},
\end{aligned}
$$

where $x_{h}: K_{h} \subset J_{h} \rightarrow \mathbf{R}^{m}$.

THEOREM 3. Let $L_{h}=p_{k}^{h} E^{k}{ }^{1} \Delta^{k}+L_{h}^{\prime}$, where $p_{k}^{h}: X_{h}^{\prime} \rightarrow X_{h}^{\prime}$ and $L_{h}^{\prime}: X_{h} \rightarrow X_{h}^{\prime}$ are linear operators satisfying

(i) there exists $C>0$ and $N \in \mathbf{N}$ such that

$$
\left|L_{h}^{\prime} x_{h}(t)\right| \leqslant C\left\|x_{h}\right\|_{k-1,[t-N h, t+N h] \cap J_{h}} \quad \forall t \in J_{h}^{\prime}, x_{h} \in X_{h}, h \in H,
$$

(ii) for some $\sigma \in(0,1)$ and with $e$ and $\bar{e}$ as defined by (14) the operator $p_{k}^{h}$ is \|\|$_{e^{-s t a b l e} \text { and } \|} \|_{\bar{e}^{-s t a b l e}}$, i.e. for some constant $C>0$ and for almost every $h \in H$

$$
\left\|x_{h}\right\|_{e} \leqslant C\left\|p_{k}^{h} x_{h}\right\|_{e} \text { and }\left\|x_{h}\right\|_{\bar{e}} \leqslant C\left\|p_{k}^{h} x_{h}\right\|_{\bar{e}} \text { for all } x_{h} \in X_{h}^{\prime} .
$$

Then the equations (12) and (13) have uniquely determined solutions $z_{h}^{a}$ and $z_{h}^{b}$, for which the estimates

$$
\left\|z_{h}^{a}\right\|_{\bar{e}} \leqslant C h^{k}\left\|y_{h}^{a}\right\|_{0}, \quad\left\|z_{h}^{b}\right\|_{e} \leqslant C h^{k}\left\|y_{h}^{b}\right\|_{0}
$$

hold. In particular, condition (V) is satisfied.

Proof. We only consider the equation (13) since (12) can be treated analogously. For grid functions $x_{h}:\left\{a_{h}, \ldots, b_{h}-\nu h\right\} \rightarrow \mathbf{R}^{m}(\nu \in\{0, \ldots, k-1\})$ we define the summation operator $\Sigma$ by 
(16) $\left(\sum x_{h}\right)\left(a_{h}\right)=0, \quad\left(\sum x_{h}\right)(t)=h \sum_{\substack{\tau \in J_{h} \\ \tau \leqslant t-h}} x_{h}(\tau)$ for $t=a_{h}+h, \ldots, b_{h}-(\nu-1) h$. $y_{h}=\Sigma x_{h}$ is the solution of the initial-value problem $\Delta y_{h}=x_{h}, y_{h}\left(a_{h}\right)=0$.

By the given representation of $L_{h}$, the equation (13) may be written in the equivalent "integral form"

$$
\left(p_{k}^{h}+L_{h}^{\prime} \Sigma^{k} E^{k}\right) v_{h}=y_{h}^{b}, \quad v_{h} \in X_{h}^{\prime}, \quad z_{h}^{b}=\Sigma^{k} E^{k} v_{h} \in X_{h} .
$$

Using (i), (ii) and (16) a straightforward calculation shows that

$$
\left\|\left(p_{k}^{h}\right)^{-1}\right\|_{e} \leqslant C, \quad\left\|\Sigma^{k} E^{k^{1}}\right\|_{e} \leqslant C h^{k}, \quad\left\|L_{h}^{\prime}\right\|_{e} \leqslant C h^{-k+1},
$$

where \|\|$_{e}$ denotes the usual operator norm. Hence $\left\|L_{h}^{\prime} \Sigma^{k} E^{k_{1}}\right\|_{e} \leqslant C h$, and an application of the Banach Lemma (cf. [11, V2.6]) shows that the operators $p_{k}^{h}+L_{h}^{\prime} \Sigma^{k} E^{k_{1}}: X_{h}^{\prime} \rightarrow X_{h}^{\prime}$ have uniformly bounded inverses (with respect to \|\|$_{e}$ ) for sufficiently small $h \in H$. Therefore, (17) has a unique solution $v_{h}$, and $z_{h}^{b}=\Sigma^{k} E^{k}{ }^{k} v_{h}$ satisfies

$$
\left\|z_{h}^{b}\right\|_{e} \leqslant C h^{k}\left\|v_{h}\right\|_{e} \leqslant C h^{k}\left\|y_{h}^{b}\right\|_{e} \leqslant C \sigma^{-n} b h^{k}\left\|y_{h}^{b}\right\|_{0} .
$$

Finally, (V) is an easy consequence of (15) and the inequalities

$$
\left\|x_{h}\right\|_{0} \leqslant C\left\|x_{h}\right\|_{e}, \quad\left\|x_{h}\right\|_{0} \leqslant C\left\|x_{h}\right\|_{\bar{e}} \quad \forall x_{h} \in X_{h}, h \in H \text {. Q.E.D. }
$$

Remark. The local condition (i) is slightly stronger than the \|\|$_{k-1}$-boundedness of $L_{h}^{\prime}$, but it will still be satisfied for any reasonable difference approximation to $L^{\prime}=\sum_{j=0}^{k-1} p_{j} D^{j}$.

It is interesting to note that the weighted stability inequality (10) can be made more precise under the assumptions of Theorem 3 if the \|\|$_{\mu}$-boundedness of $R_{h}$ is replaced by the more detailed condition

$$
\left\|R_{h} x_{h}\right\|_{0} \leqslant C\left(\left\|x_{h}\right\|_{\mu_{a},\left[a_{h}, a_{h}+\widetilde{N} h\right] \cap J_{h}}+\left\|x_{h}\right\|_{\mu_{b},\left[b_{h}-\tilde{N} h, b_{h}\right] \cap J_{h}}\right)
$$

for all $x_{h} \in X_{h}, h \in H$ and for some $\mu_{a}, \mu_{b} \in\{0, \ldots, k-1\}$ and $\widetilde{N} \in \mathbf{N}$ independent of $x_{h}$ and $h$.

Normally (18) will be satisfied if $R$ describes two-point boundary conditions with orders $\mu_{a}$ in $a$ and $\mu_{b}$ in $b$.

Corollary. Suppose that $\left(L_{h}, R_{h}\right)$ is \|\|$_{k}$-stable and that the assumptions of Theorem 3 hold. If $R_{h}$ satisfies (18) then the stability inequality

$$
\begin{aligned}
&\left\|x_{h}\right\|_{j} \leqslant C\left(\left\|L_{h} x_{h}\right\|_{0, J_{h}^{0}}+h^{k-\mathrm{Max}\left(\mu_{a}, j\right)}\left\|L_{h} x_{h}\right\|_{0, J_{h}^{a}}\right. \\
&\left.+h^{k-\mathrm{Max}\left(\mu_{b}, j\right)}\left\|L_{h} x_{h}\right\|_{0, J_{h}^{b}}+\left\|R_{h} x_{h}\right\|_{0}\right) \quad(j=0, \ldots, k)
\end{aligned}
$$

is valid for all $x_{h} \in X_{h}$, almost every $h \in H$ and $J_{h}^{a}=\left\{a_{h}^{\prime}, \ldots, a_{h}^{\prime}+n_{a} h\right\}, J_{h}^{b}=$ $\left\{b_{h}^{\prime}-n_{b} h, \ldots, b_{h}^{\prime}\right\}, J_{h}^{0}=J_{h}^{\prime} \backslash\left(J_{h}^{a} \cup J_{h}^{b}\right)$.

The proof makes use of the inequalities (15) but, apart from this, is quite similar to that of Theorem 1 and will, therefore, be omitted. 
The "local effect" of inequality (19) in comparison to (10) will be illustrated in Section 5 for our example (5), (6).

4. The Root Conditions. In Section 3 we have reduced the two main assumptions of Theorem 1-\| $\|_{k}$-stability of $\left(L_{h}, R_{h}\right)$ and condition (V)-to the question of whether $p_{k}^{h}$ is stable with respect to the norms \|\|$_{0},\|\|_{e}$ and \|\|$_{\bar{e}}$.

In what follows we shall prove that the answer is affirmative in any case under the same general conditions, which will be called the root conditions.

For that purpose we assume a special structure for the operator $p_{k}^{h}$. Let $p_{k}(t)=$

$[a, b]$ and suppose that the difference approximation of $D^{k}$ in $J_{h}^{\prime}$ results from the application of the same difference formula (usually of higher order) at all inner grid points, and a finite number of formulas (usually of lower order or asymmetric) near the boundary (cf. [1] , [2], [8]). Taking into account the fact that we can factor $\Delta^{k}$ out of these formulas (see [17], [1]), we arrive at the following representation:

$$
p_{k}^{h} x_{h}(t)= \begin{cases}\left(q_{j}(E) x_{h}\right)\left(a_{h}^{\prime}\right) & \text { for } t=a_{h}^{\prime}+j h, j=0, \ldots, \alpha-1, \\ \left(P(E) x_{h}\right)(t) & \text { for } t=a_{h}^{\prime}+\alpha h, \ldots, b_{h}^{\prime}-\bar{\alpha} h \\ \left(\bar{q}_{j}\left(E^{-1}\right) x_{h}\right)\left(b_{h}^{\prime}\right) & \text { for } t=b_{h}^{\prime}-j h, j=0, \ldots, \bar{\alpha}-1,\end{cases}
$$

where $P(E)=\Sigma_{i=-\alpha}^{\bar{\alpha}} \xi_{i} E^{i}(\alpha, \bar{\alpha} \in \mathbf{N})$ and $q_{j}, j=0, \ldots, \alpha-1$, and $\bar{q}_{j}, j=0, \ldots, \bar{\alpha}-1$, are real polynomials independent of $h$. Equation (20) was introduced and investigated by Grigorieff [8] in his analysis of stability with respect to Sobolev norms. For our example (5), (6) it is clear that $p_{2}^{h}$ is of the form (20) (see [1] for further examples).

Let $\sigma_{i} \in \mathbf{C}, i=1, \ldots, d$, and $\bar{\sigma}_{j} \in \mathbf{C}, j=1, \ldots, \bar{d}$, denote the distinct roots of $z^{\alpha} P(z)$ and $z^{\bar{\alpha}} P\left(z^{-1}\right)$, respectively, which lie inside the unit circle, and let $v_{i}$ and $\bar{v}_{j}$ be the corresponding multiplicities. Then we have

THEOREM 4. Let $p_{k}^{h}: X_{h}^{\prime} \rightarrow X_{h}^{\prime}$ be of the form (20). Then $p_{k}^{h}$ is \|\|$_{0}$-stable if and only if the following root conditions hold:

(R1) $P(z) \neq 0$ for all $z \in \mathbf{C},|z|=1$.

(R2) $\alpha=\Sigma_{i=1}^{d} v_{i}, \bar{\alpha}=\Sigma_{j=1}^{\bar{d}} \bar{v}_{j}$ and the quadratic matrices

$$
\begin{aligned}
& Q=\left(q_{i-1}^{(\nu-1)}\left(\sigma_{j}\right): \begin{array}{l}
i=1, \ldots, \alpha, \\
\nu=1, \ldots, v_{j}, j=1, \ldots, d
\end{array}\right) \text { and } \\
& \bar{Q}=\left(\bar{q}_{\bar{\alpha}-i}^{(\nu-1)}\left(\bar{\sigma}_{j}\right): \begin{array}{l}
i=1, \ldots, \bar{\alpha} \\
\nu=1, \ldots, \bar{v}_{j}, j=1, \ldots, \bar{d}
\end{array}\right)
\end{aligned}
$$

are nonsingular.

For a proof of Theorem 4 and a detailed discussion of the literature ([7], [8] , [12]) we refer to [1]. It should be noted that (R1) and (R2) are satisfied for a wide class of finite difference schemes applied to boundary value problems of even order ([1]). 
THEOREM 5. Let $p_{k}^{h}$ be of the form (20) and let the root conditions (R1) and

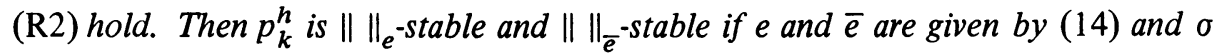
satisfies

$$
\left|\sigma_{i}\right|,\left|\bar{\sigma}_{j}\right|<\sigma<1 \quad \text { for } i=1, \ldots, \alpha \text { and } j=1, \ldots, \bar{\alpha} \text {. }
$$

Proof. Without loss of generality we may assume $m=1$ since $p_{k}^{h}$ is a "diagonal" operator, i.e., $\left(p_{k}^{h} x_{h}\right)_{i}$ only depends on $\left(x_{h}\right)_{i}$ for $i=1, \ldots, m$. Furthermore, we confine ourselves to \|\|$_{e}$-stability (the modifications necessary in the case of \|\|$_{\bar{e}}$-stability will be obvious from the proof). It will be convenient to introduce complex valued grid functions and to identify $J_{h}^{\prime}$ and $\{0, \ldots, n\}, X_{h}^{\prime}$ and $\mathbf{R}^{n+1}, p_{k}^{h}$ and the real part of the complex operator

$$
\left(p_{k, n} x\right)(j)=\left\{\begin{array}{l}
q_{j}(E) x(0), j=0, \ldots, \alpha-1, \\
P(E) x(j), j=\alpha, \ldots, n-\bar{\alpha}, \\
\bar{q}_{n-j}\left(E^{-1}\right) x(n), j=n-\bar{\alpha}+1, \ldots, n,
\end{array} \quad x \in \mathbf{C}^{n+1}, n \in \mathbf{N} .\right.
$$

Now $e(j)=\sigma^{n-j}(j=0, \ldots, n)$ and the norms \|\|$_{0},\|\|_{e}$ can be extended directly to $\mathbf{C}^{n+1}$. Our proof is divided into two steps:

S1: For every $r \in \mathbf{C}^{n+1}$ there exists $x \in \mathbf{C}^{n+1}$ such that $p_{k, n} x(j)=r(j)$ for $j=\alpha, \ldots, n-\bar{\alpha}$ and $\|x\|_{e} \leqslant C\|r\|_{e}$.

S2: For every $r \in \mathbf{C}^{n+1}$ satisfying $r(j)=0$ for $j=\alpha, \ldots, n-\bar{\alpha}$, the equation $p_{k, n} x=r$ has a unique solution $x \in \mathbf{C}^{n+1}$ and $x$ satisfies $\|x\|_{e} \leqslant C\|r\|_{e}$.

If an arbitrary $r \in \mathbf{C}^{n+1}$ is given, we determine $x$ as in S1 and solve $p_{k, n} y=$ $r-p_{k, n} x$ according to S2. Then $p_{k, n}(x+y)=r$ and

$$
\|x+y\|_{e} \leqslant\|x\|_{e}+\|y\|_{e} \leqslant C\left(\|r\|_{e}+\left\|r-p_{k, n} x\right\|_{e}\right) \leqslant C\left(\|r\|_{e}+\|x\|_{e}\right) \leqslant C\|r\|_{e} .
$$

Proof of S1. By (R1) and (R2) we have for some constant $w \in \mathrm{C}$

$$
P(E)=w E^{\bar{\alpha}-\alpha} \prod_{i=1}^{d}\left(E-\sigma_{i} I\right)^{v_{i}} \prod_{i=1}^{\bar{d}}\left(\bar{\sigma}_{i} I-E^{-1}\right)^{\bar{v}_{i}}
$$

Hence, it will be sufficient to prove that for any grid function $s:\{\eta, \ldots, n-\bar{\eta}\}$ $\rightarrow \mathbf{C}$, where $\eta, \bar{\eta} \in \mathbf{N}, \eta \leqslant \alpha, \bar{\eta} \leqslant \bar{\alpha}$, and any $\lambda \in\left\{\sigma_{i}, i=1, \ldots, d, \bar{\sigma}_{i}, i=1, \ldots, \bar{d}\right\}$, the initial value problems $(E-\lambda I) y(j)=s(j)$ for $j=\eta, \ldots, n-\bar{\eta}, y(\eta)=0$ and $\left(\lambda I-E^{-1}\right) \bar{y}(j)=s(j)$ for $j=\eta, \ldots, n-\bar{\eta}, \bar{y}(n-\bar{\eta})=0$ have solutions $y$ and $\bar{y}$ satisfying $\|y\|_{e},\|\bar{y}\|_{e} \leqslant C\|s\|_{e}$. But this follows easily from (21) and the well-known formulas

$$
y(j)=\sum_{\nu=\eta}^{j-1} \lambda^{j-1-\nu} s(\nu) \text { and } \bar{y}(j)=-\sum_{\nu=j+1}^{n-\bar{n}} \lambda^{\nu-j-1} s(\nu) .
$$

Proof of S2. Suppose $r \in \mathbf{C}^{n+1}$ satisfies $r(j)=0$ for $j=\alpha, \ldots, n-\bar{\alpha}$. Then any solution $x \in \mathbf{C}^{n+1}$ of $p_{k . n} x=r$ is a fundamental solution of the difference oper- 
ator $P(E)$ and, therefore, can be represented as

$$
x=\sum_{\nu, j} \gamma_{\nu j} \varphi_{\nu j}^{n}+\sum_{\nu, j} \bar{\gamma}_{\nu j} \bar{\varphi}_{\nu j}^{n}
$$

where

$$
\begin{aligned}
& \varphi_{\nu j}^{n}(i)=\left(\prod_{\mu=i-\nu+2}^{i} \mu\right) \sigma_{j}^{i-\nu+1}, \quad v=1, \ldots, v_{j}, j=1, \ldots, d(i=0, \ldots, n), \\
& \bar{\varphi}_{\nu j}^{n}(i)=\left(\prod_{\mu=n-i-\nu+2}^{n-i} \mu\right) \bar{\sigma}_{j}^{n-i-\nu+1}, \quad v=1, \ldots, \bar{v}_{j}, j=1, \ldots, \bar{d}(i=0, \ldots, n),
\end{aligned}
$$

form a basis of the null space of $P(E)$ (cf. [10]).

Substituting (22) into the equation $p_{k, n} x=r$ we arrive at the following $(\alpha+\bar{\alpha}) \times(\alpha+\bar{\alpha})$ linear system (cf. [1])

$$
\left(\begin{array}{cc}
Q & Q_{n} \\
\bar{Q}_{n} & \bar{Q}
\end{array}\right)\left(\begin{array}{l}
\gamma \\
\bar{\gamma}
\end{array}\right)=\left(\begin{array}{l}
r^{0} \\
r^{1}
\end{array}\right),
$$

where $r^{0}=(r(0), \ldots, r(\alpha)), r^{1}=(r(n-\bar{\alpha}), \ldots, r(n)), \gamma=\left(\gamma_{\nu j}: \nu=1, \ldots, v_{j}\right.$, $j=1, \ldots, d) \in \mathbf{C}^{\alpha}, \bar{\gamma}=\left(\bar{\gamma}_{\nu j}: \nu=1, \ldots, \bar{v}_{j}, j=1, \ldots, \bar{d}\right) \in \mathbf{C}^{\bar{\alpha}} . Q$ and $\bar{Q}$ are the quadratic matrices defined in (R2) and $Q_{n}$ and $\bar{Q}_{n}$ are $\alpha \times \bar{\alpha}$ and $\bar{\alpha} \times \alpha$ matrices satisfying $\left\|Q_{n}\right\|_{0},\left\|\bar{Q}_{n}\right\|_{0} \leqslant C \sigma^{n}$.

By (R2) the matrices

$$
\left(\begin{array}{cc}
A_{n} & B_{n} \\
C_{n} & D_{n}
\end{array}\right)=\left(\begin{array}{cc}
Q & Q_{n} \\
\bar{Q}_{n} & \bar{Q}
\end{array}\right)^{-1}
$$

exist for sufficiently large $n$ and are uniformly bounded with respect to \|\|$_{0}$ (cf. [1]). Hence, (23) has a unique solution $\left(\frac{\gamma}{\gamma}\right)$ and the corresponding $x \in \mathbf{C}^{n+1}$, as defined by (22), satisfies $p_{k, n} x=r$ and

$$
\|x\|_{e} \leqslant C\left(\|\gamma\|_{0} \sum_{\nu, j}\left\|\varphi_{\nu j}^{n}\right\|_{e}+\|\bar{\gamma}\|_{0} \sum_{\nu, j}\left\|\bar{\varphi}_{\nu j}^{n}\right\|_{e}\right)
$$

Our proof is finished by the application of the following estimates:

$$
\begin{aligned}
\left\|\bar{\varphi}_{\nu j}^{n}\right\|_{e} & \leqslant C \quad \text { for } \nu=1, \ldots, \bar{v}_{j}, j=1, \ldots, \bar{d}, \\
\left\|\varphi_{\nu j}^{n}\right\|_{e} & \leqslant C \sigma^{-n} \text { for } \nu=1, \ldots, v_{j}, j=1, \ldots, d, \\
\|\bar{\gamma}\|_{0} & \leqslant\left\|\left(\begin{array}{c}
\gamma \\
\bar{\gamma}
\end{array}\right)\right\|_{0} \leqslant C\left\|\left(\begin{array}{c}
r^{0} \\
r^{1}
\end{array}\right)\right\|_{0}=C\|r\|_{0} \leqslant C\|r\|_{e} \text { and } \\
\|\gamma\|_{0} & =\left\|A_{n} r^{0}+B_{n} r^{1}\right\|_{0} \leqslant\left\|A_{n}\right\|_{0}\left\|r^{0}\right\|_{0}+\left\|A_{n} Q_{n} \bar{Q}^{-1}\right\|_{0}\left\|r^{1}\right\|_{0} \\
& \leqslant C \sigma^{n-\alpha+1}\|r\|_{e}+C\left\|Q_{n}\right\|_{0}\|r\|_{e} \leqslant C \sigma^{n}\|r\|_{e} \text {. Q.E.D. }
\end{aligned}
$$


Summarizing Theorems $1-5$, we obtain the following result:

Consider a system (1) of linear boundary value problems of order $k$ with the associated pair $\left(L=D^{k}+L^{\prime}, R\right)$, and let a sequence of approximating linear operators ( $L_{h}=p_{k}^{h} E^{-k} \Delta^{k}+L_{h}^{\prime}, R_{h}$ ) be given such that the following conditions hold:

(i) 0 is not an eigenvalue of $(L, R)$,

(ii) $p_{k}^{h}$ is of the form (20) and satisfies the root conditions (R1), (R2) and the consistency relations $q_{j}(1)=1(j=0, \ldots, \alpha-1), P(1)=1, \bar{q}_{j}(1)=1(j=0, \ldots, \bar{\alpha}-1)$,

(iii) $L_{h}^{\prime}$ is consistent with $L^{\prime}$ and "locally \|\|$_{k-1}$-bounded", i.e., statisfies condition (i) of Theorem 3,

(iv) $R_{h}$ is consistent with $R$ and \|\|$_{\mu}$-bounded for some $\mu \in\{0, \ldots, k\}$.

Then the weighted stability inequality (10) holds for $j=0, \ldots, k$ with $J_{h}^{1}=$ $\left\{a_{h}^{\prime}, \ldots, a_{h}^{\prime}+n_{a} h, b_{h}^{\prime}-n_{b} h, \ldots, b_{h}^{\prime}\right\}, J_{h}^{0}=\left\{a_{h}^{\prime}+\left(n_{a}+1\right) h, \ldots, b_{h}^{\prime}-\left(n_{b}+1\right) h\right\}$ where $n_{a}, n_{b}$ are arbitrary fixed integers. If, in addition, $R_{h}$ satisfies (18) then the sharper inequality (19) is valid.

5. Numerical Results. Based on the inequality (19) we can now generalize the rule of Bramble and Hubbard as follows:

Let $L$ be of order $k$ and let $R$ describe two-point boundary conditions with orders $\mu_{a}$ and $\mu_{b}$ in $a$ and $b$, respectively. Further, let $D_{h}^{j}: X_{h} \rightarrow X_{h}^{\prime}$ be \|\|$_{j}$-bounded linear operators $(0 \leqslant j \leqslant k)$. Assume for the true solution $\bar{x}$ of (1) that

$L_{h}$ is consistent with $L$ of order $\tau_{a}$ in $J_{h}^{a}, \tau$ in $J_{h}^{0}$ and $\tau_{b}$ in $J_{h}^{b}$,

$R_{h}$ is consistent with $R$ of order $\rho$ and

$D_{h}^{j}$ is consistent with $D^{j}$ of order $\delta$.

Let $\bar{x}_{h}$ denote the solution of (2) with $r_{h}=[r]_{h}^{\prime}$, then the global discretization error $\epsilon_{j}(h):=\left\|\left[\bar{x}^{(j)}\right]_{h}^{\prime}-D_{h}^{j} \bar{x}_{h}\right\|_{0}$ satisfies

$$
\begin{gathered}
\epsilon_{j}(h)=O\left(h^{M}\right), \\
M=\operatorname{Min}\left(\tau, \rho, \delta, \tau_{a}+k-\operatorname{Max}\left(j, \mu_{a}\right), \tau_{b}+k-\operatorname{Max}\left(j, \mu_{b}\right)\right) .
\end{gathered}
$$

This is an easy consequence of (19) and the inequality

$$
\left\|\left[\bar{x}^{(j)}\right]_{h}^{\prime}-D_{h}^{j} \bar{x}_{h}\right\|_{0} \leqslant\left\|\left[\bar{x}^{(j)}\right]_{h}^{\prime}-D_{h}^{j}[\bar{x}]_{h}\right\|_{0}+C\left\|[\bar{x}]_{h}-\bar{x}_{h}\right\|_{j}
$$

We note that (24) already follows from the stability inequality (10) if $\tau_{a}=\tau_{b}$.

In order to obtain $O\left(h^{\tau}\right)$-convergence with respect to \|\|$_{0}$ it is, therefore, sufficient to choose $\rho=\delta=\tau, \tau_{a}=\tau-k+\mu_{a}, \tau_{b}=\tau-k+\mu_{b}$. This choice leads to

$$
\epsilon_{j}(h)=\left\{\begin{array}{l}
O\left(h^{\tau}\right) \text { for } 0 \leqslant j \leqslant \mu_{0}:=\operatorname{Min}\left(\mu_{a}, \mu_{b}\right), \\
O\left(h^{\tau-\left(j-\mu_{0}\right)}\right) \text { for } \mu_{0}<j \leqslant k
\end{array}\right.
$$

For our example (5), (6) we took $[a, b]=[0,1], p_{0}(t)=-4 t, r(t)=4 \sin (2 t)-$ $4\left(1-t^{2}\right) \cos (2 t), \bar{x}(t)=(1-t) \cos (2 t)$. The results are displayed in the following table: 


\begin{tabular}{ccccccc}
\hline$h$ & $\epsilon_{0}(h)$ & $\operatorname{Ord}_{0}(h)$ & $\epsilon_{1}(h)$ & $\operatorname{Ord}_{1}(h)$ & $\epsilon_{2}(h)$ & $\operatorname{Ord}_{2}(h)$ \\
\hline$\frac{1}{10}$ & $5.95 \mathrm{E}-4$ & 3.001 & $9.37 \mathrm{E}-4$ & 2.707 & $2.29 \mathrm{E}-2$ & 2.064 \\
$\frac{1}{20}$ & $7.43 \mathrm{E}-5$ & 2.998 & $1.43 \mathrm{E}-4$ & 2.904 & $5.49 \mathrm{E}-3$ & 2.034 \\
$\frac{1}{40}$ & $9.30 \mathrm{E}-6$ & 2.997 & $1.91 \mathrm{E}-5$ & 2.962 & $1.34 \mathrm{E}-3$ & 2.013 \\
$\frac{1}{80}$ & $1.16 \mathrm{E}-6$ & 2.998 & $2.46 \mathrm{E}-6$ & 2.983 & $3.30 \mathrm{E}-4$ & 2.009 \\
\hline
\end{tabular}

The quotient $\operatorname{Ord}_{j}(h)=\log \left(\epsilon_{j}(h) / \epsilon_{j}(h / 2)\right) / \log 2$ has been used to estimate the order of convergence (cf. [13]). (24) is applicable with $k=2, \mu_{a}=1, \mu_{b}=0, \tau_{a}=2, \tau=4$, $\tau_{b}=2, \rho=3, \delta=4$ and yields the orders 3,3 and 2 for $j=0,1$ and 2 . These were reproduced very accurately by the numerical computation.

We also considered a modification of the difference equations (5), (6) where a third order formula was used for the differential equation at $t=a$ and a fourth-order formula was used to discretize the boundary condition $x^{\prime}(a)=\gamma_{a}$. This corresponds to $\tau_{a}=3$ and $\rho=4$, and by (24) we then have $\epsilon_{0}(h)=O\left(h^{4}\right), \epsilon_{1}(h)=O\left(h^{3}\right), \epsilon_{2}(h)$ $=O\left(h^{2}\right)$. Note that in this case the application of the stability inequality (10) (with $\mu=1$ ) only yields $\epsilon_{0}(h)=O\left(h^{3}\right)$ because of the $O\left(h^{2}\right)$-formula used at $t=b-h$.

The numerical results are

\begin{tabular}{cccc}
\hline$h$ & $\operatorname{Ord}_{0}(h)$ & $\operatorname{Ord}_{1}(h)$ & $\operatorname{Ord}_{2}(h)$ \\
\hline$\frac{1}{10}$ & 4.047 & 2.834 & 2.066 \\
$\frac{1}{20}$ & 4.033 & 2.967 & 2.036 \\
$\frac{1}{40}$ & 4.019 & 2.994 & 2.019 \\
$\frac{1}{80}$ & 4.010 & 3.000 & 2.009 \\
\hline
\end{tabular}

Finally, we treated the fourth-order problem

$$
x^{\mathrm{IV}}+(\pi-t) x=r \quad \text { in }[0, \pi], \quad x(0)=x(\pi)=x^{\prime \prime}(\pi)=0, \quad x^{\prime \prime}(0)=-4 \pi,
$$

where $r$ was determined in such a way that $x(t)=(\pi-t)^{2} \sin (t)$ is the true solution. For our finite difference method we took $h=\pi N^{-1}, N \geqslant 10, a_{h}=-h, b_{h}=\pi+h$, $k_{1}=k_{2}=2$ and used appropriate difference formulas with the orders of consistency $\tau_{a}=\tau_{b}=2, \tau=4, \rho=4, \delta=4$. 


\begin{tabular}{cccccc}
\hline$h$ & $\operatorname{Ord}_{0}(h)$ & $\operatorname{Ord}_{1}(h)$ & $\operatorname{Ord}_{2}(h)$ & $\operatorname{Ord}_{3}(h)$ & $\operatorname{Ord}_{4}(h)$ \\
\hline$\frac{\pi}{10}$ & 3.20 & 3.41 & 3.42 & 3.50 & 1.61 \\
$\frac{\pi}{20}$ & 3.72 & 3.72 & 3.67 & 2.16 & 1.89 \\
$\frac{\pi}{40}$ & 3.89 & 3.87 & 3.84 & 2.72 & 1.95 \\
$\frac{\pi}{80}$ & 3.91 & 3.92 & 3.92 & 2.88 & 1.97 \\
\hline by & 4 & 4 & 4 & 3 & 2 \\
\hline
\end{tabular}

For the same differential equation with the boundary conditions $x(0)=x(\pi)=x^{\prime}(\pi)$ $=0, x^{\prime}(0)=\pi^{2}$ we used a discretization leading to the orders of consistency $\tau_{a}=\tau_{b}$ $=2, \tau=6, \rho=6, \delta=6$ if $0 \leqslant j \leqslant 2$ and $\delta=4$ if $j=3,4$ (see [1] for a proof of the root conditions). Now $\mu_{a}=\mu_{b}=1, k-\mu_{a}=3$ and by (24) the expected orders are $\operatorname{Ord}_{0}(h)=\operatorname{Ord}_{\mathbf{i}}(h)=5, \operatorname{Ord}_{2}(h)=4, \operatorname{Ord}_{3}(h)=3, \operatorname{Ord}_{4}(h)=2$. The numerical results are contained in the following table.

\begin{tabular}{cccccc}
\hline$h$ & $\operatorname{Ord}_{0}(h)$ & $\operatorname{Ord}_{1}(h)$ & $\operatorname{Ord}_{2}(h)$ & $\operatorname{Ord}_{3}(h)$ & $\operatorname{Ord}_{4}(h)$ \\
\hline$\frac{\pi}{10}$ & 3.92 & 3.76 & 4.04 & 3.06 & 1.61 \\
$\frac{\pi}{20}$ & 4.67 & 4.33 & 4.60 & 2.53 & 1.89 \\
$\frac{\pi}{4.0}$ & 4.86 & 4.71 & 4.76 & 2.83 & 1.95 \\
$\frac{\pi}{80}$ & 4.76 & 4.84 & 4.97 & 2.93 & 1.97 \\
\hline
\end{tabular}

We note that the orders of convergence predicted by (24) are already attained approximately for larger values of $h$ (with the exception of $\operatorname{Ord}_{2}(h)$, which seems to be underestimated by (24)). This is important since for smaller values of $h$ the exact order of convergence cannot be observed due to the rounding errors occurring in the direct solution of the system (2). For fourth-order problems numerical experiments show that these rounding errors become crucial at least if $h \leqslant 1 / 100$ provided the calculation is done in a precision of 16 decimal digits.

6. Cptimal Weighted Stability Inequalities. Our numerical results suggest that the weighted stability inequality $(10)$ in connection with the given orders of consistency determines the exact order of convergence (at least if $\tau_{a}=\tau_{b}$ and $j \leqslant \mu$ ). Indeed, we shall prove that inequality (10) is sharp in a certain sense. More precisely, the exponent $k-\mu$ in (10) (for $j=0$ ) cannot be increased by 1 if $R_{h}$ is \|\|$_{\mu}$-bounded 
but not \|\|$_{\mu-1}$-bounded. As in Section 2 we consider a boundary value problem (1) and linear operators $L_{h}: X_{h} \rightarrow X_{h}^{\prime}, R_{h}: X_{h} \rightarrow \mathbf{R}^{m k}(h \in H)$.

THEOREM 6. Let $\left(L_{h}, R_{h}\right)$ be consistent with $(L, R)$ and let $L_{h}$ and $R_{h}$ be \|\|$_{k}$-bounded. Let a series of subsets $J_{h}^{0} \subset J_{h}^{\prime}$ and $N_{a}, N_{b} \in \mathbf{N}, \mu \in\{0, \ldots, k-1\}$ be given such that $N_{a}, N_{b} \geqslant \mu$ and

$$
\begin{gathered}
R_{h} x_{h}=0 \quad \text { if } x_{h}=0 \text { in }\left\{a_{h}, \ldots, a_{h}+N_{a} h, b_{h}-N_{b} h, \ldots, b_{h}\right\}, \\
L_{h} x_{h}=0 \text { in } J_{h}^{0} \text { if } x_{h}=0 \text { in }\left\{a_{h}+N_{a} h, \ldots, b_{h}-N_{b} h\right\} .
\end{gathered}
$$

If the weighted stability inequality

$$
\left\|x_{h}\right\|_{0} \leqslant C\left(\left\|L_{h} x_{h}\right\|_{0, J_{h}^{0}}+h^{k-\mu}\left\|L_{h} x_{h}\right\|_{0, J_{h}^{1}}+\left\|R_{h} x_{h}\right\|_{0}\right),
$$

where $J_{h}^{1}=J_{h}^{\prime} \backslash J_{h}^{0}$, holds for all $x_{h} \in X_{h}$ and almost every $h \in H$, then $R_{h}$ is \|\|$_{\mu^{-}}$ bounded.

Remarks. 1. The conditions (25) and (26) require that the boundary conditions and the difference equations in $J_{h}^{0}$ (which usually consists of interior grid points) form an uncoupled system. This is a natural assumption for difference approximations to two-point boundary value problems.

2. It is interesting to note that we assume inequality (10) only for $j=0$. Hence, Theorem 6 also applies to difference schemes $\left(L_{h}, R_{h}\right)$ for which weighted stability inequalities have been derived by means of inverse monotonicity (cf. [2] , [14]).

Proof. Let us assume to the contrary that there exists a subsequence $H^{\prime} \subset H$ and a sequence $x_{h} \in X_{h}\left(h \in H^{\prime}\right)$ such that $\left\|R_{h} x_{h}\right\|_{0}=1,\left\|x_{h}\right\|_{\mu} \rightarrow 0\left(h \in H^{\prime}\right)$. Let $M=\operatorname{Max}(k, 2(\mu+1))$ and consider the difference equations

$$
\begin{aligned}
& \Delta^{M} y_{h}(t)=0 \text { for } t=a_{h}, \ldots, b_{h}-M h, \\
& \Delta^{j} y_{h}(t)=\Delta^{j} x_{h}(t) \quad \text { for } t=a_{h}+\left(N_{a}-\mu\right) h, b_{h}-N_{b} h \text { and } j=0, \ldots, \mu, \\
& \Delta^{j} y_{h}\left(a_{h}+\left(N_{a}-\mu\right) h\right)=0 \text { for } j=\mu+1, \ldots, M-\mu-2 .
\end{aligned}
$$

These form a consistent approximation to the boundary value problem $y^{(M)}=0$ in $[a, b], y^{(j)}(a)=0$ for $j=0, \ldots, M-\mu-2, y^{(j)}(b)=0$ for $j=0, \ldots, \mu$, which has only the trivial solution. Applying Theorem 2 with $k=M, k_{1}=0, p_{M}^{h}=I$, we obtain \|\|$_{M}$-stability and $\left\|y_{h}\right\|_{k} \leqslant\left\|y_{h}\right\|_{M} \leqslant C\left\|x_{h}\right\|_{\mu} \rightarrow 0$. Now $v_{h}:=x_{h}-y_{h}$ satisfies $\left\|R_{h} v_{h}\right\|_{0} \rightarrow 1,\left\|v_{h}\right\|_{\mu} \rightarrow 0\left(h \in H^{\prime}\right)$ and

(28) $v_{h}=0$ in $\left\{a_{h}+\left(N_{a}-\mu\right) h, \ldots, a_{h}+N_{a} h, b_{h}-N_{b} h, \ldots, b_{h}-\left(N_{b}-\mu\right) h\right\}$.

Let $w_{h}$ result from $v_{h}$ by setting $v_{h}$ to 0 in $\left\{a_{h}+\left(N_{a}+1\right) h, \ldots, b_{h}-\left(N_{b}+1\right) h\right\}$; then by (25), (26) and (28) we have $R_{h} w_{h}=R_{h} v_{h}, L_{h} w_{h}=0$ in $J_{h}^{0},\left\|w_{h}\right\|_{\mu} \rightarrow 0$ ( $h \in H^{\prime}$ ). According to (27), the system

$$
L_{h} z_{h}=0 \quad \text { in } J_{h}^{\prime}, \quad R_{h} z_{h}=R_{h} w_{h},
$$


has a unique solution $z_{h} \in X_{h}$ for which it holds that

$$
\left\|z_{h}-w_{h}\right\|_{0} \leqslant C h^{k-\mu}\left\|L_{h} w_{h}\right\|_{0, J_{h}^{1}} \leqslant C h^{k-\mu}\left\|w_{h}\right\|_{k} \leqslant C\left\|w_{h}\right\|_{\mu} \rightarrow 0
$$

and, hence, $\left\|z_{h}\right\|_{0} \rightarrow 0\left(h \in H^{\prime}\right)$. Since $\left\|R_{h} w_{h}\right\|_{0} \rightarrow 1\left(h \in H^{\prime}\right)$, there exists a $g \in \mathbf{R}^{m k}, g \neq 0$, and a subsequence $H^{\prime \prime} \subset H^{\prime}$ such that $R_{h} w_{h} \rightarrow g\left(h \in H^{\prime \prime}\right)$. Now it follows easily from the \|\|$_{0}$-stability of $\left(L_{h}, R_{h}\right)$ and its consistency with $(L, R)$ that 0 is not an eigenvalue of $(L, R)$. Therefore, the boundary value problem $L z=0$ in $[A, B], R z=g$ has a unique solution $z \in\left(C^{k}[A, B]\right)^{m}$ and $\left\|[z]_{h}-z_{h}\right\|_{0} \rightarrow 0$ ( $h \in H^{\prime \prime}$ ), since (29) is a consistent and \|\|$_{0}$-stable approximation to this problem. Finally, $\left\|z_{h}\right\|_{0} \rightarrow 0$ yields $z=0$ in $[a, b]$ and $R z=0$, which contradicts $g \neq 0$. Q.E.D.

It is obvious from the proof that the condition (26) can be weakened to $L_{h} x_{h}=0$ in $J_{h}^{0}$ if $x_{h}=0$ in $\left\{a_{h}+\left(N_{a}-\mu\right) h, \ldots, b_{h}-\left(N_{b}-\mu\right) h\right\}$.

Furthermore, we remark that we can establish a full equivalence between the \|\|$_{\mu}$-boundedness of $R_{h}$ and the condition that the weighted stability inequality (27) is valid for $J_{h}^{1}=\left\{a_{h}, \ldots, a_{h}+n_{a} h, b_{h}-n_{b} h, \ldots, b_{h}\right\}$ and any fixed $n_{a}, n_{b} \in \mathbf{N}$ (with $C$ depending on $n_{a}, n_{b}$ ). For that purpose we have to assume that the conditions (i), (ii) and (iii) at the end of Section 4 hold and that $R_{h}$ is consistent with $R$ and satisfies a local estimate

$$
\left\|_{i} R_{h} x_{h}\right\|_{0} \leqslant C\left(\left\|x_{h}\right\|_{k,\left[a_{h}, a_{h}+\bar{N} h\right] \cap J_{h}}+\left\|x_{h}\right\|_{k,\left[b_{h}-\bar{N} h, b_{h}\right] \cap J_{h}}\right)
$$

for some $\bar{N} \in N$ independent of $h$ and $x_{h}$.

The conditions (25) and (26) will then be satisfied for appropriate $N_{a}, N_{b} \in \mathbf{N}$.

Institut für Numerische und instrumentelle Mathematik

Universität Münster

Roxeler Strasse 64

D-4400 Münster, West Germany

1. W.-J. BEYN, “Zur Stabilität von Differenzenverfahren für Systeme linearer gewöhnlicher Randwertaufgaben," Numer. Math., v. 29, 1978, pp. 209-226.

2. E. BOHL, Zur Anwendung von Differenzenschemen mit symmetrischen Formeln bei Randwertaufgaben, ISNM 32, Birkhäuser Verlag, Berlin, 1976, pp. 25-47.

3. J. H. BRAMBLE \& B. E. HUBBARD, "On the formulation of finite difference analogues of the Dirichlet problem for Poisson's equation," Numer. Math., v. 4, 1962, pp. 313-327.

4. J. H. BRAMBLE \& B. E. HUBBARD, "On a finite difference analogue of an elliptic boundary problem which is neither diagonally dominant nor of non-negative type," J. Math. and Phys., v. 43, 1964, pp. 117-132.

5. J. H. BRAMBLE \& B. E. HUBBARD, "New monotone type approximations for elliptic problems," Math. Comp., v. 18, 1964, pp. 349-367.

6. P. G. CIARLET, "Discrete maximum principle for finite-difference operators," Aequationes Math., v. 4, 1970, pp. 338-352.

7. H. ESSER, "Stabilitätsungleichungen für Diskretisierungen von Randwertaufgaben gewöhnlicher Differentialgleichungen," Numer. Math., v. 28, 1977, pp. 69-100.

8. R. D. GRIGORIEFF, “Die Konvergenz des Rand- und Eigenwertproblems linearer gewöhnlicher Differenzengleichungen," Numer. Math., v. 15, 1970, pp. 15-48.

9. N. N. GUDOVICH, "A new method for constructing stable finite-difference schemes of any previously assigned order of approximation for linear ordinary differential equations," U.S.S.R. Computational Math. and Math. Phys., v. 15 (4), 1975, pp. 115-129.

10. P. HENRICI, Discrete Variable Methods in Ordinary Differential Equations, Wiley, New York, London, Sydney, 1962. 
11. L. W. KANTOROWITSCH \& G. P. AKILOW, Funktionalanalysis in normierten Räumen, Akademie Verlag, Berlin, 1964.

12. H.-O. KREISS, "Difference approximations for boundary and eigenvalue problems for ordinary differential equations," Math. Comp., v. 26, 1972, pp. 605-624.

13. J. LORENZ, Die Inversmonotonie von Matrizen und ihre Anwendung beim Stabilitätsnachweis von Differenzenverfahren, Ph. D. Thesis, University of Münster, 1975.

14. J. LORENZ, "Zur Inversmonotonie diskreter Probleme," Numer. Math., v. 27, 197.7, pp. $227-238$.

15. H. S. PRICE, "Monotone and oscillation matrices applied to finite difference approximations," Math. Comp., v. 22, 1968, pp. 489-516.

16. H. J. STETTER, Analysis of Discretization Methods for Ordinary Differential Equations, Springer Tracts in Natural Philosophy, Vol. 23, Springer-Verlag, Berlin, Heide!berg, New York, 1973.

17. V. THOMÉE \& B. WESTERGREN, "Elliptic difference equations and interior regularity," Numer. Math., v. 11, 1968, pp. 196-210. 